Original Research Paper

\title{
Computational Analysis of Network ODE Systems in Metric Spaces: An Approach
}

\author{
Susmit Bagchi \\ Department of Aerospace and Software Engineering (Informatics), \\ Gyeongsang National University, Jinju, Republic of Korea
}

Article history

Received: 02-01-2017

Revised: 24-02-2017

Accepted: 28-02-2017

Email: profsbagchi@gmail.com

\begin{abstract}
Traditionally, the concepts of graph theory are applied to design stationary computer networks and, to analyze dynamics of social networks. However, the majority of non-stationary network models are formulated by using Ordinary Differential Equations (ODE) with varying orders having homogeneous or non-homogeneous forms. However, the analysis of continuous solution spaces of ODE and understanding of the interplay of spaces in complex systems are difficult to formulate. This paper proposes an analytical model and an algorithm to analyze as well as determine characteristics of solution spaces of system of ODE. The analytical model employs structural elements of metric spaces. The algorithmic output and analysis illustrate that, the proposed model successfully determines the dynamics of solution intervals as well as structural interactions.
\end{abstract}

Keywords: Networks, Ordinary Differential Equations, Metric Spaces, Predicate

\section{Introduction}

The applications of graph theoretic models as well as structures of networking are pervasive in nature encompassing computer networks, social networks and biochemical networks. Traditionally, the modeling and analysis of stationary networked systems consider graph algorithmic approaches. However, every network systems are not stationary in nature. The networked systems can be broadly classified into two domains namely, stationary class and non-stationary class. The wireless networks of computers and social network systems are prime examples of non-stationary and highly dynamic networked systems (Tipper and Sundareshan, 1990). The data traffic in computer networks resemblances the characteristics of periodic processes with oscillation (Medykovsky et al., 2013). It is observed that, the dynamics of network flows exhibit the existence of stochastic elements and can be modeled as discrete event dynamical systems (Obaidat et al., 2015). In general, the modeling and analysis of complex network dynamics follow two broad approaches. In one approach, the network dynamics is considered to be nonstationary in nature. In other approaches, the concept of randomized Boolean functional network is employed (Ridden and MacArthur, 2012). In biochemical systems, the Boolean networks are derived through various transformations (Stotzel et al., 2015). The wireless networks are highly non-stationary in nature having lower stability as compared to wired networks. The analysis of dynamics of wireless networks requires the applications of Markov models (Bylina et al., 2012). However, in majority of cases the models of complex networks are formulated by employing Ordinary Differential Equations (ODE) having constant or varying coefficients as well as homogeneity. For example, the dynamics of computer networks are modeled by employing first order ODE (Medykovsky et al., 2013). The models of complex networks assume continuous time varying dynamics, which are often equipped with smooth functions. The applications of ODE imply that dynamics of complex networks are derived in continuous solution spaces. On the other hand, the stochastic discrete event based network modeling with quantized states indicates that, non-stationary dynamics of complex networks can be formulated in discrete domains having deterministic solution intervals.

\section{Motivation}

The modeling and analysis of complex networks are formulated by using first order ODE in linear or nonlinear forms having varying coefficients. The homogeneity of ODE intended for modeling complex networks is dependent on the characteristics of networks. The solution spaces of such models are continuous and 
may have large intervals. In case of complex systems modeled with system of ODE, the continuous solution spaces may have complex structures spreading over large intervals. The determination of characteristics and interplay of system of ODE becomes difficult if the continuous solution spaces are non-linear in nature having close interactions. The analysis of solution spaces of system of ODE can be facilitated by the applications of structures of metric spaces. The pairwise comparison of decomposed solution spaces helps in determining the characteristics of solution intervals and understanding of structural interplays. In line with the approach of employing metric spaces, the algorithmic analysis may be conducted on sampled data sets from solution spaces to compute and represent solution intervals. This paper proposes the computational analysis of solution spaces of system of ODE by following concepts of metric spaces. The main contributions of this paper are as follows:

- Formulating a model for analyzing solution spaces of system of ODE in metric spaces

- Designing an algorithm for sampled data analysis to determine solution intervals and interactions

- Determining a set of axioms to transform continuous spaces into discrete forms for computational analysis

Rest of the paper is organized as follows. The second section describes related work in the field. Third section presents the proposed analytical model. Next, fourth section presents the algorithm and the computational evaluations. Lastly, fifth section concludes the paper.

\section{Related Work}

The applications of network and associated graph models are pervasive in nature encompassing computer networks, social networks and biochemical networks. The dynamics and control of computer networks are considered to be non-stationary in nature. In general, the computer network systems are modeled by employing concepts of queuing theory. However, the Ordinary Differential Equations (ODE) based model of computer networks is formulated considering non-stationary conditions (Tipper and Sundareshan, 1990). The model considers combinations of queuing theory and, nonlinear ODE. Researchers have proposed that, the dynamics of computer networks can be modeled by employing first order ODE and Ateb-functions (Medykovsky et al., 2013). The computer network flow analysis and modeling follow the characteristics of discrete event based dynamical systems. In general, the discrete event based modeling and formalisms (DEVS) are used to represent discrete dynamical systems
(Obaidat et al., 2015). However, the computer network analysis often requires incorporation of stochastic parameters. Thus, the stochastic DEVS formalisms are employed to model and analyze computer networks, which utilize the Quantized State Systems (QSS) (Obaidat et al., 2015). The QSS-based network analysis requires approximated ODE in order to represent the networked systems.

The applications of ODE are found in network modeling and analysis in biological systems (Minerva et al., 2015). The ODE based network analysis is used to predict and suppress network-inflation of cells. The random Boolean network based analysis is employed to understand gene regulation and activation (Ridden and MacArthur, 2012). In this network, the size of the network is very large and, each node is connected to a set of randomly selected nodes with assigned Boolean functions. The analysis of network dynamics of random and large-scale Boolean network also requires the applications of ODE (Ridden and MacArthur, 2012). The transient and steady-state conditions in complex networks can be analyzed by formulating ODE (Soliman and Heiner, 2010). In modeling large-scale network systems with randomness, the transformation of such network into Boolean networks is required. Researchers have proposed to analyze ODE based networked system analysis deriving from Euler-like transformations (Stotzel et al., 2015). The appearances of transients in wireless networks are observable phenomena. The transient analysis of wireless sensor networks requires probabilistic models. The modeling and analysis of transient probabilities in wireless sensor networks is formulated by employing first order ODE (Bylina et al., 2012). The ODE-based model of network transient probabilities follows matrix-vector form.

The generation of solutions of system of ODE with reduced errors requires large computing capacities and often employs neural network techniques (Meade Jr. and Fernandez, 1994; Lagaris et al., 1998). In general highperformance distributed computing systems are employed to execute ODE solvers (Burrage and Pohl, 1994). The decomposed solvers execute on distributed nodes connected by network and the decomposed modules communicate over network. Researchers have proposed solvers of higher order ODE by using radial basis functions (Mai-Duy, 2005). The major challenge in different types of solvers is to determine the interplay of solutions of set of ODE. In general, such analysis is performed graphically after the execution of the solvers. However, the data sets generated by solvers are very large and are often in the range of GB. Thus, data analysis becomes an error prone and complex task. An analytical model is required to predict and analyze characteristics of solutions and their interplay in solution spaces. The modeling and predicting information 
diffusion in social network is an interesting research topic. The information diffusion model in networked systems is formulated using ODE and is employed to understand the dynamics of network structures as well as sizes. In general, the ODE-based epidemiological model is used to analyze information diffusion in large networks (Hethcote, 2000; Yang and Leskovec, 2010). The analysis of information diffusion in social networks is conducted by using logistic equation in the form of first order ODE (Davoudi and Chatterjee, 2016). In another approach, the epidemic model based information diffusion in network is formulated considering heterogeneous network environments (Stai et al., 2015). Furthermore, the explicit network diffusion model is employed to study malware attacks in wireless networks (Khouzani et al., 2012).

\section{Analytical Model}

The formulation of analytical model requires transformation of continuous solution spaces of a system of ODE into the discrete domain. In that direction, first a set of basic concepts is presented. Next, the detailed analytical framework is presented in axiomatic representations.

\section{Basic Definitions}

In this section, the construction of analytical solution spaces and its transformation into discrete domain is formulated by considering a set of ODE in general form. Let $\psi=\{[a, b]: a, b \in \mathfrak{R}\}$ be a set of intervals. The set of $n$ th order differential equations in general form is given by a system of ODE in matrix representation as:

$$
\begin{aligned}
& {\left[a(x)_{i j}\right]\left[\begin{array}{ll}
D^{n} y & D^{n-1} y \ldots . . y
\end{array}\right]^{T}=\left[r_{i}(x)\right]^{T}} \\
& 1<i \leq N, a(x)_{i 1}=1
\end{aligned}
$$

The differential equations may have constant or varying coefficients as well as can be homogeneous or non-homogeneous in nature. If the analytical solutions of differential equations of Equation 1 are $y \mathrm{I}=\left[h_{i}\left(x, r_{i}\right.\right.$ $(x))]^{T}$ where I is unit column matrix, then there exist corresponding predicates $P_{i}(x, y) \in\{0,1\}$ signifying the analytical solutions in discrete domain represented as:

$$
\left[P_{i}(x, y)=1\right] \Rightarrow\left[y-h_{i}\left(x, r_{i}(x)\right)=0\right]
$$

The predicate indicates that, the evaluation to Boolean true value follows the solutions of differential equations in analytical form. Let, the solution interval of $P_{i}(x, y)$ be $X_{i} \in \psi$. The mapping within the solution spaces of differential equations is given by, $f: \psi \rightarrow(Y \subset \mathfrak{R})$ such that, $\forall y \in Y: y=f(x \in \mathfrak{R})$. The set of solutions of differential equations in solution spaces are computed by corresponding binary relations having conditions as given below:

$$
\left[R_{i} \subset X_{i} \times Y\right] \Rightarrow R_{i}=\left\{(x, y): P_{i}(x, y)\right\}
$$

The formal model considers that the relations are anti-symmetric and non-transitive in nature.

\section{Transformation and Analysis}

Suppose, $(A, d)$ be a metric space where, $A \subseteq \bigcup_{k=1}^{N} X_{k} \times Y$ and, $d: A^{2} \rightarrow \Re$ such that:

$$
\begin{aligned}
& d\left(A^{2}\right)=\sqrt{(\Delta x)^{2}+(\Delta y)^{2}} \\
& d\left(A^{2}\right) \in[0,+\infty) \\
& d\left(\left(x_{1}, y_{1}\right),\left(x_{3}, y_{3}\right)\right) \leq \\
& d\left(\left(x_{1}, y_{1}\right),\left(x_{2}, y_{2}\right)\right)+d\left(\left(x_{2}, y_{2}\right),\left(x_{3}, y_{3}\right)\right)
\end{aligned}
$$

The boundary evaluation conditions of respective metric spaces are governed by following axioms:

$$
\begin{aligned}
& \exists[a, b] \in \psi:[a, b]=X_{i} \cap X_{k}, \\
& {\left[d\left(A^{2}\right)=0\right] \Rightarrow[\Delta x=0 \wedge \Delta y=0] \vee} \\
& {\left[\forall x \in[a, b]: P_{i}(x, f(x)) \wedge P_{k}(x, f(x))\right]}
\end{aligned}
$$

According to the axioms, the distance metric can be reduced to zero if and only if the corresponding solution spaces have identical solutions. There must be an interval of such identical solutions and, the solution spaces outside of such interval may not be comparable. Furthermore, the interplay between solution spaces of a system of ODE may generate three cases.

\section{Case I: Solution Spaces are Pair-Wise Disjoint}

In this case, the solution spaces of system of ODE are considered to be pair-wise disjoint in nature. The identification of such solution spaces can be formulated by following axiom:

$$
\left[R_{i} \cap R_{k}=\phi\right] \Rightarrow \neg\left[\exists(x, y) \in A: P_{i}(x, y) \wedge P_{k}(x, y)\right]
$$

This dynamics of solution spaces indicate that, there exist no common general as well as particular solutions of the differential equations in solution spaces in analytical forms. Hence, the set of distance metric in metric spaces can be computed as: 


$$
S_{d}\left[P_{i}, P_{k}\right]=\left\{d\left(R_{i} \times R_{k}\right): d(.)>0\right\}
$$

The maximum and minimum separations between solution spaces of two differential equations are computed as, $\max \left(S_{d}\left[P_{i}, P_{k}\right]\right)$ and, $\min \left(S_{d}\left[P_{i}, P_{k}\right]\right)$ respectively. Furthermore, if in a system $\max \left(S_{d}\left[P_{i}\right.\right.$, $\left.\left.P_{k}\right]\right)=\min \left(S_{d}\left[P_{i}, P_{k}\right]\right)$, then the corresponding solution spaces are equidistant everywhere for the respective ODE pair.

Case II: Solution Spaces are Pair-Wise Nondisjoint

In this case, the pairs of ODE in a system have the solution spaces in pair-wise non-disjoint forms, where all solutions are not identical. The axiomatic representation of such solution spaces can be given by:

$$
\left[R_{i} \cap R_{k} \neq \phi\right] \Rightarrow\left[\exists(x, y) \in A: P_{i}(x, y) \wedge P_{k}(x, y)\right]
$$

Hence, the pairs of ODE have at least one common solution in respective solution spaces. Suppose, $\exists i$ : $\exists k \neq$ $i$ : $1<k \leq N$ such that $\left|R_{i} \cap R_{k}\right|>1$ indicating there exist multiple common solutions in the respective solution spaces. The natures of such common solutions can be determined by following three conditions.

\section{Condition C1: Existence of Multiple Discrete Common Solutions}

The two differential equations may contain multiple discrete common solutions if following axiom is satisfied:

$$
\begin{aligned}
& A_{i k}=R_{i} \cap R_{k},\left|A_{i k}\right|>1, \\
& \left|R_{i}\right|+\left|R_{k}\right|>\left|A_{i k}\right|, \\
& \forall\left(x_{c}, y_{c}\right) \forall\left(x_{d}, y_{d}\right) \in A_{i k}: \\
& {[\Delta x \neq 0 \vee \Delta y \neq 0] \Rightarrow\left[d\left(A_{i k}{ }^{2}\right)>0\right]}
\end{aligned}
$$

The axiom illustrates that, the solution spaces do not have any continuous intervals of common solutions. However, discrete points of converging solutions exist in solution spaces. Furthermore, if $B=\left\{d\left(A_{i k}{ }^{2}\right):\left[A_{i k}=R_{i} \cap\right.\right.$ $\left.\left.R_{k}\right] \wedge\left[d\left(A_{i k}{ }^{2}\right)>0\right]\right\}$, then the extremals of divergences in separation in solution spaces is given by:

$$
\partial B=|\sup (B)-\inf (B)|
$$

\section{Condition C2: Existence of Region of Continuous Common Solutions}

It is possible that, the solution spaces of a system of ODE have an interval of continuous common solutions. The pairs of ODE in a system have a region of continuous common solutions if the following axiom is satisfied:

$$
\begin{aligned}
& \exists[a, b] \in \psi: \forall x \in[a, b]: A_{i k}=\{(x, f(x))\},\left|A_{i k}\right|>1, \\
& \left|R_{i}\right|+\left|R_{k}\right|>\left|A_{i k}\right|, \\
& {\left[A_{i k}=R_{i} \cap R_{k}\right] \Rightarrow\left[d\left(A_{i k}^{2}\right)=0\right]}
\end{aligned}
$$

It is important to note that, the common solution interval is continuous in nature. The solution spaces outside of the interval are disjoint.

\section{Condition C3: Existence of Multiple Intervals of Continuous Common Solutions}

This is an extension of condition $C 2$. The system of ODE may have multiple intervals of common solutions with continuity if the following axiom is satisfied:

$$
\begin{aligned}
& \psi_{A} \subset \psi, \\
& \forall[a, b] \in \psi_{A}: \forall x \in[a, b]: A_{i k}=\{(x, f(x))\},\left|A_{i k}\right|>1, \\
& \left|R_{i} \cup R_{k}\right| \geq\left|A_{i k}\right|, \\
& {\left[A_{i k}=R_{i} \cap R_{k}\right] \Rightarrow\left[d\left(A_{i k}{ }^{2}\right)=0 \wedge\left|\psi_{A}\right|>1\right]}
\end{aligned}
$$

Thus, the there exists a set of common solutions within multiple continuous intervals within the solution spaces. The intervals between common solution spaces may be disjoint. The solutions of ODE outside of these intervals are disjoint in nature iff $\left|R_{i} \cup R_{k}\right|>\left|A_{i k}\right|$. The solutions spaces are identical iff $\left|R_{i} \cup R_{k}\right|=\left|A_{i k}\right|$.

\section{Case III: Solution Spaces are in Globally Non- Disjoint Intervals}

In this case, the axiomatic conditions for obtaining globally non-disjoint intervals of common solutions of system of ODE in solution spaces are constructed. The existence of globally non-disjoint intervals of solutions indicates that the system has at least one common solution in original solution spaces. The axioms of determining such global intervals are given as:

$$
\begin{aligned}
& \psi_{A} \subset \psi, m \geq 1, \\
& \forall[a, b] \in \psi_{A}: \forall x \in[a, b]: A=\{(x, f(x))\},|A|>1, \\
& \left|\bigcup_{k=1}^{N} R_{k}\right| \geq|A|, \\
& {\left[A=\bigcap_{k=1}^{N} R_{k}\right] \Rightarrow\left[d\left(A^{2}\right)=0 \wedge\left|\psi_{A}\right|=m\right]}
\end{aligned}
$$

The multiplicity of regions of common solutions depends on value of $m$. If $m=1$ then there is a unique 
region of common solution for the given system of ODE. Otherwise, $m>1$ signifies the existence of multiplicity of such regions in original solution spaces.

\section{Computational Evaluations}

The computational evaluations of the proposed analytical model are conducted in order to formulate geometric representation of the characteristics of solution spaces of a system of ODE. Following the general forms of ODE-based models of computer networks, a system of ODE is considered to be comprised of mixed first order linear and non-linear homogeneous as well as non-homogeneous equations as presented below:

$$
\begin{aligned}
& (D y)^{2}-x D y+y=0, \\
& D y=k y, \\
& D y-y=e^{x}, \\
& D y-y=y^{2}, \\
& x D y=-y
\end{aligned}
$$

The solution spaces are analyzed in metric spaces by executing computational algorithm on sampled data sets from the continuous solution spaces. The pseudo-code representation of the algorithm is illustrated in Fig. 1.

The algorithm implements pair-wise comparison of solution spaces of system of ODE under transformation in metric spaces. Initial values of Boolean flags indicating existence of disjoint solution intervals (dint) and common intervals (cint) are set to false. The sets of disjoint solution intervals (dis) and common solution intervals (intv) in solution spaces are marked as empty. The algorithm compares and sorts the pair-wise solution spaces until the data sets are exhausted. Next, the predicate function is evaluated on sorted disjoint intervals in order to compute the intervals of common solution intervals. The predicate function considers interval length $(\mathrm{w})$ for comparison to be fixed at 0.3 . If the common solution intervals exist in the solution spaces of system of ODE, then the intervals are aggregated and the existence of common solution intervals are indicated in the respective flag.

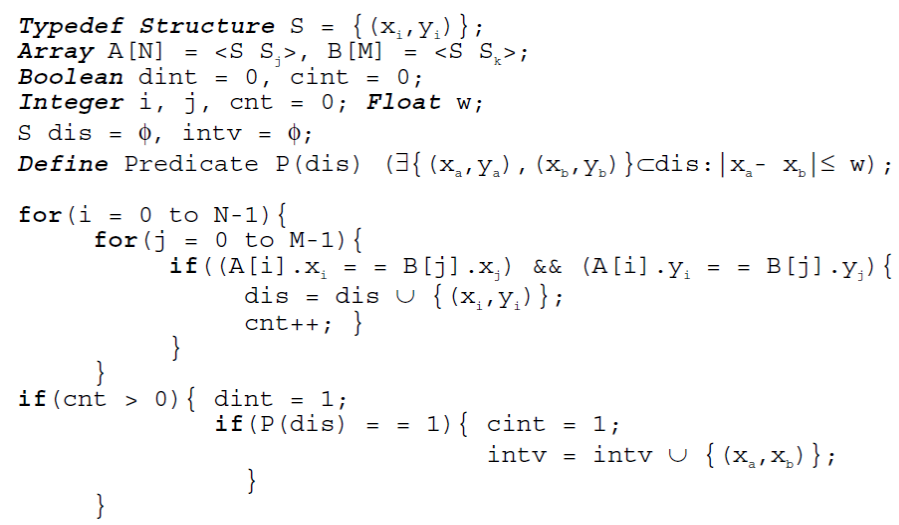

Fig. 1. Pseudo-code representation of algorithm

Continuous solution spaces of system of ODE

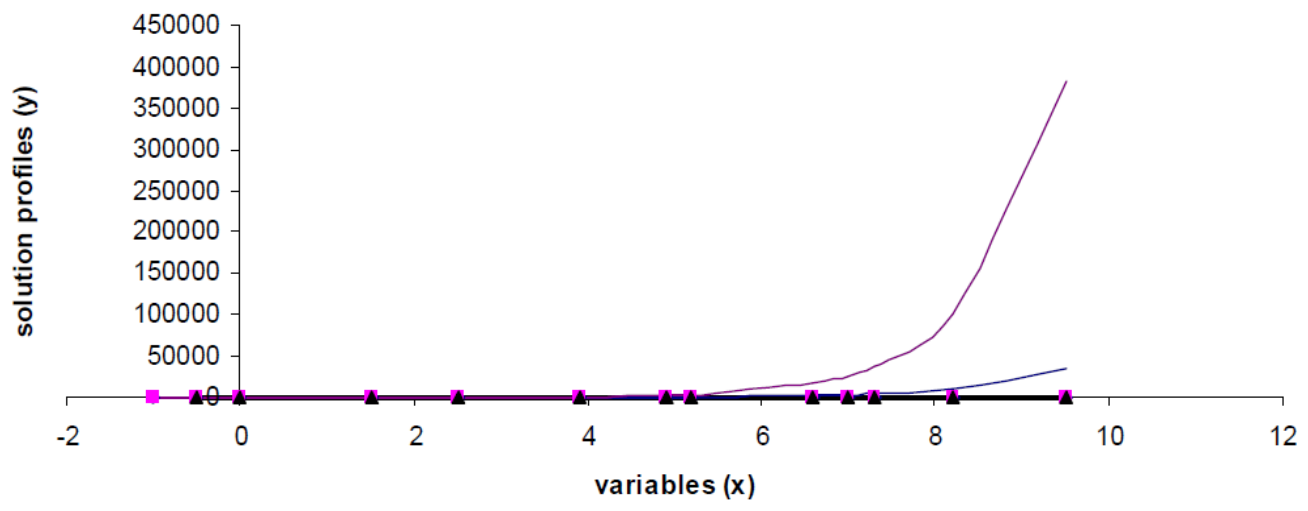

Fig. 2. Continuous solution spaces of system of ODE in 2-D 


\section{Continuous solution spaces of ODE system (Subset I)}

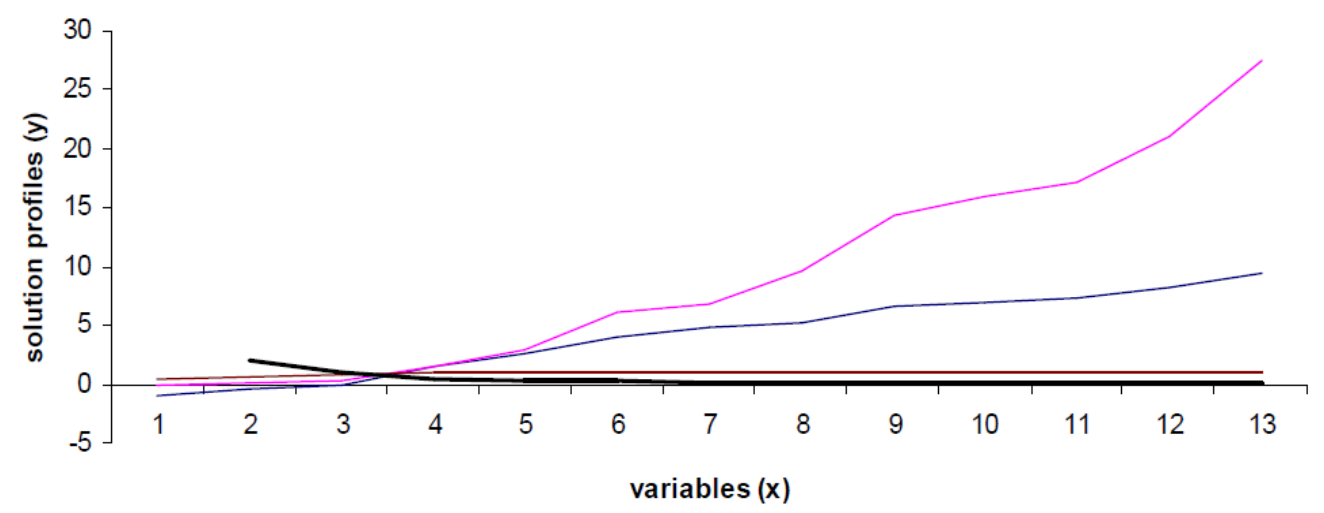

Fig. 3. Continuous solution spaces of ODE system-reduced dimension (LGF)

\section{Continuous solution spaces of system of ODE (Subset II)}

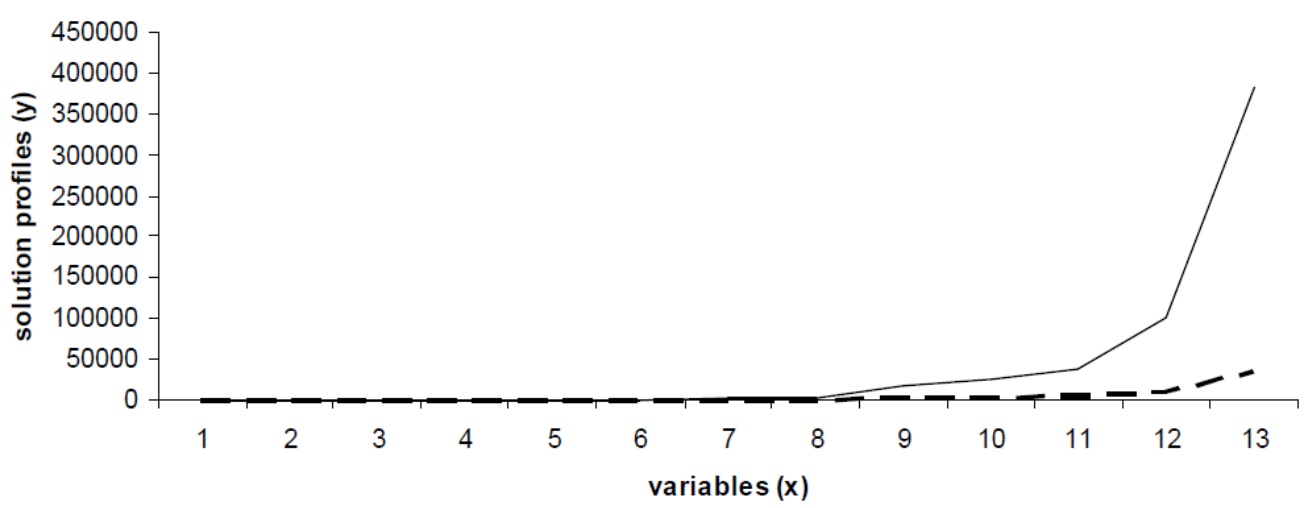

Fig. 4. Continuous solution spaces of ODE system-reduced dimension (HGF)

The dynamics of continuous solution spaces of system of ODE in 2-D plane are illustrated in Fig. 2. It can be observed in Fig. 2 that, the intervals of disjoint and non-disjoint solutions are indistinguishable due to the mixed growth factors of individual solution spaces.

The dynamics of continuous solution spaces of subset of ODE (Subset I) having low growth factors (LGF) are illustrated in Fig. 3 in 2-D plane.

Figure 3 illustrates that, accurate system dynamics are more distinguishable as compared to Fig. 2 due to the reduction of dimension of the system. Similar effect is observable in Fig. 4 illustrating continuous solution spaces in 2-D plane of a system having High Growth Factor (HGF) in solution dynamics with reduced dimension.

Figure 4 illustrates that, the computed solutions in continuous solution spaces are convergent at lower values of variables ( $\mathrm{x}$ ) and, tend to diverge if $\mathrm{x}$ increases monotonically.

\section{Algorithmic Analysis}

The computational analysis of data sets is conducted by the algorithm in order to detect disjoint and nondisjoint solution intervals of the system under consideration. The analysis is performed by algorithm using pair-wise solution spaces in three sets (Set I, II and III). Set I considers pair-wise system of ODE having solution spaces with LGF. Set II considers pair-wise system of ODE with moderate growth factor in solution spaces. Lastly, Set III considers a pair of ODE with HGF. The computed distance metric in solution spaces and associated contours of solution intervals for Set I are presented in Fig. 5 and 6, respectively. The computed distance metric and associated contours of solution intervals for Set II are presented in Fig. 7 and 8, respectively. The corresponding results for Set III are illustrated in Fig. 9 and 10. The computed contours of solution intervals in metric spaces have successfully identified the disjoint and non-disjoint intervals of 
solution spaces of the system under consideration. In case of Set I, the existence of common solution intervals (non-disjoint) is identified, where the interval is larger than contours of Set II and Set III.

Distance metric in solution spaces (pair-wise)- Set I

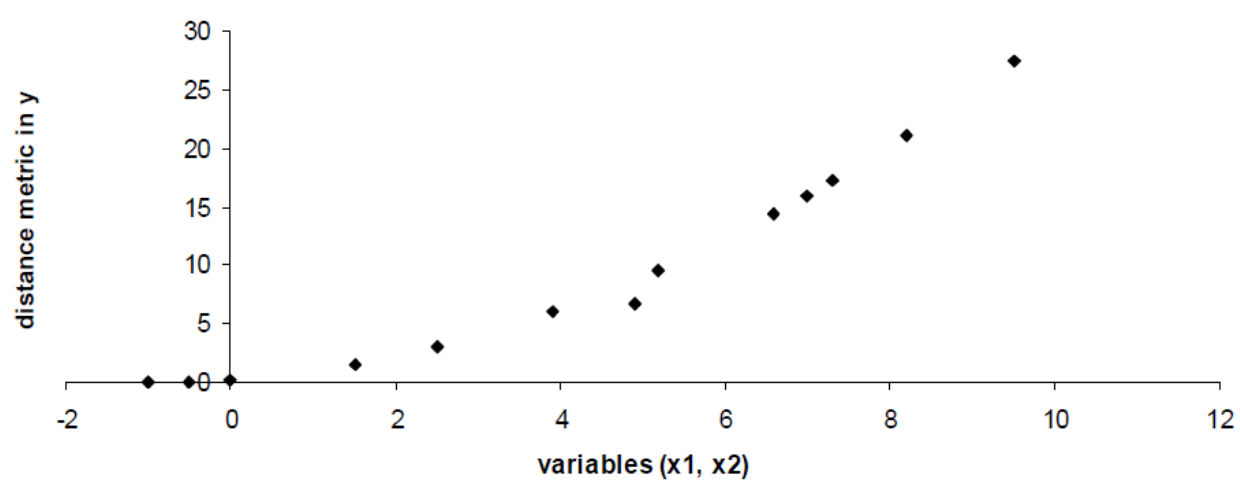

Fig. 5. Computed distance metric for Set I

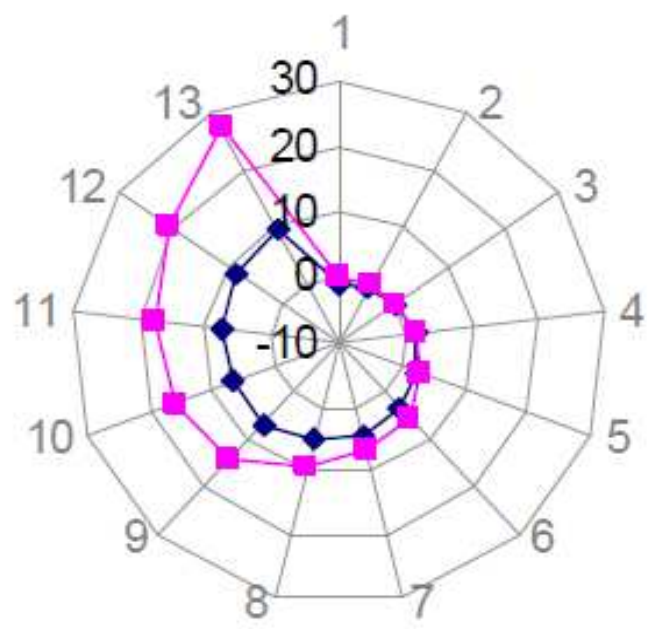

Fig. 6. Contours of solution intervals for Set I

\section{Distance metric in solution spaces (pair-wise)-Set II}

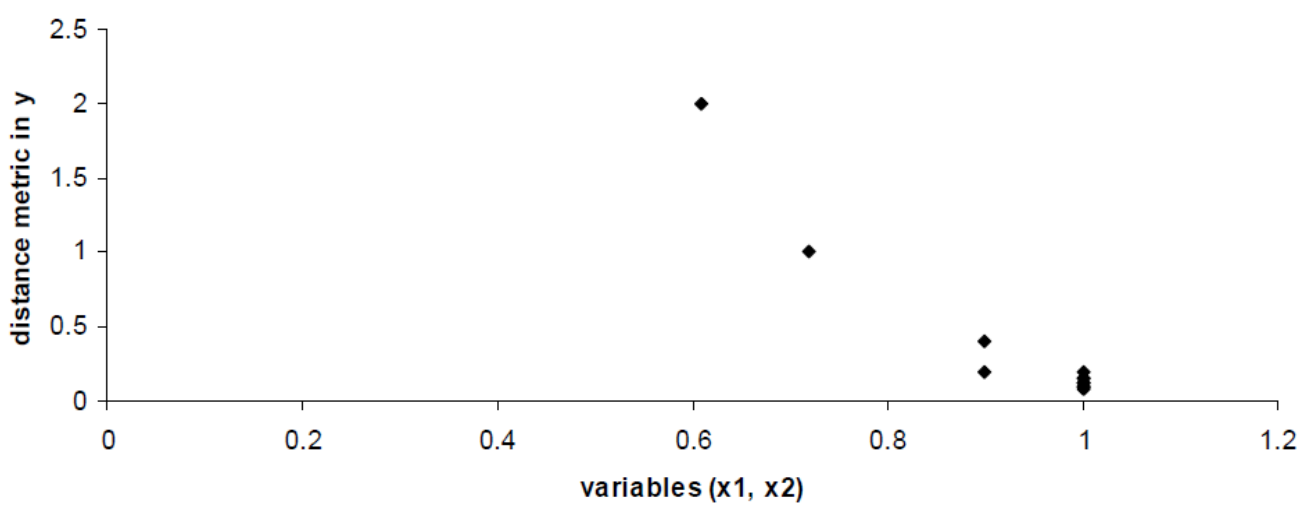

Fig. 7. Computed distance metric for Set II 


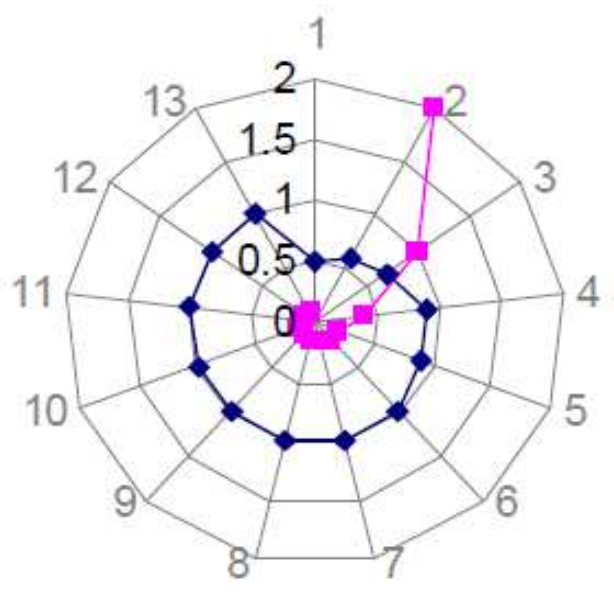

Fig. 8. Contours of solution intervals for Set II

\section{Distance metric in solution spaces (pair-wise)-Set III}

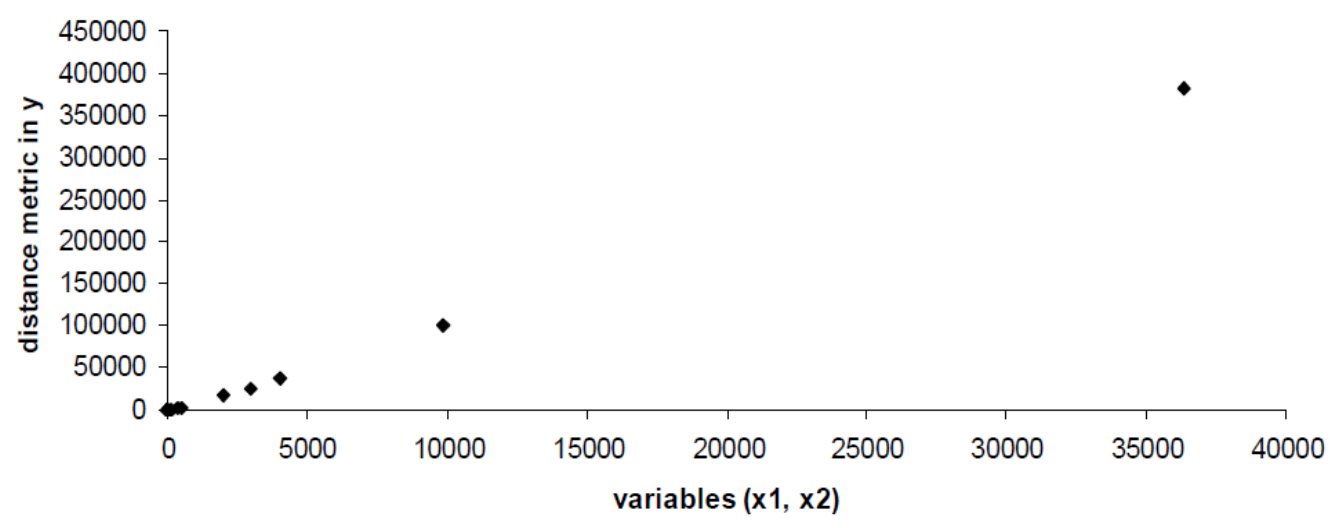

Fig. 9. Computed distance metric for Set III

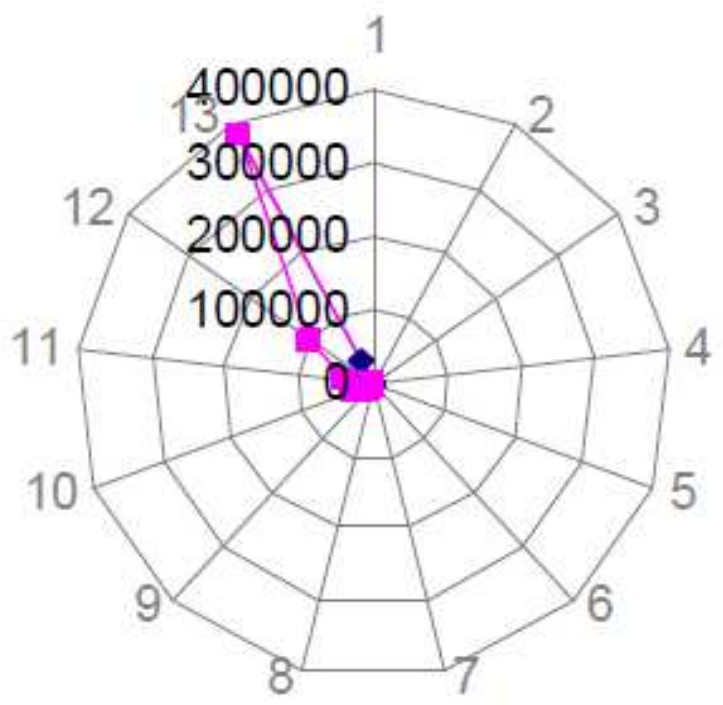

Fig. 10. Contours of solution intervals for Set III 
The contours of distance metric in solution spaces for Set II indicate that, there exist multiple discrete common solutions and the solution spaces are largely disjoint in nature. However, in case of Set III, the common solution intervals are concentrated in a region and, the solution spaces are disjoint outside of the region. Moreover, the region of disjoint solution intervals is larger than the common solution interval.

\section{Conclusion}

The applications of networking structures and models are found in computer systems, social networking systems and biochemical network systems. In majority of cases, the network modeling requires employment of ODE. The analysis of characteristics of solution spaces of system of ODE having mixed forms and orders is difficult in continuous multidimensional spaces. The application of metric spaces in analysis helps in determining the interplay of solution spaces of ODE systems. The algorithmic analysis by considering discrete samples from solution spaces can effectively uncover the existence of common solution intervals, nature of disjoint intervals and multiplicity of common solution intervals. The applications of metric spaces and corresponding algorithmic analysis of solution spaces of system of ODE facilitates the determination and understanding of interplay of solution spaces in complex systems.

\section{Ethics}

This article is original contribution of the author and is not published elsewhere. There is no ethical issue involved in this article.

\section{References}

Bylina, B., M. Karwacki and J. Bylina, 2012. A CPUGPU hybrid approach to the uniformization method for solving Markovian models-A case study of a wireless network. Proceedings of the 19th International Conference on Computer Networks, Jun. 19-23, Springer, Szczyrk, Poland, pp: 401-410. DOI: $10.1007 / 978-3-642-31217-5 \_42$

Burrage, K. and B. Pohl, 1994. Implementing an ODE code on distributed memory computers. Comput. Math. Applic., 28: 10-12.

DOI: 10.1016/0898-1221(94)00194-4

Davoudi, A. and M. Chatterjee, 2016. Prediction of information diffusion in social networks using dynamic carrying capacity. Proceedings of the IEEE International Conference on Big Data, Dec. 5-8, IEEE Xplore Press, pp: 2466-2469.

DOI: $10.1109 /$ BigData.2016.7840883
Hethcote, H.W., 2000. Recent advances in information diffusion and influence maximization of complex social networks. SIAM Rev.

Khouzani, M.H.R., S. Sarkar and E. Altman, 2012. Maximum damage malware attack in mobile wireless networks. IEEE/ACM Trans. Network., 20: $1347-1360$.

DOI: $10.1109 /$ TNET.2012.2183642

Lagaris, I.E., A. Likas and D.I. Fotiadis, 1998. Artificial neural networks for solving ordinary and partial differential equations. IEEE Trans. Neural Netw., 9: 987-1000.

DOI: $10.1109 / 72.712178$

Medykovsky, M., I. Droniuk, M. Nazarkevich and O. Fedevych, 2013. Modelling the pertubation of traffic based on ateb-functions. Proceedings of the 20th International Conference on Computer Networks, Jun. 17-21, Springer, Poland, pp: 38-44. DOI: $10.1007 / 978-3-642-38865-1$ 5

Minerva, D., S. Kawasaki and T. Suzuki, 2015. Pathway network analysis and an application to the ODE model of MMP2 activation in the early stage of cancer cell invasion. AIP Conf. Proc., 1651: 99-99. DOI: $10.1063 / 1.4914439$

Meade Jr. A.J. and A.A. Fernandez, 1994. The numerical solution of linear ordinary differential equations by feedforward neural networks. Math. Comput. Model., 19: 1-25.

DOI: 10.1016/0895-7177(94)90095-7

Mai-Duy, N., 2005. Solving high order ordinary differential equations with radial basis function networks. Int. J. Numer. Meth. Eng., 62: 824-852. DOI: $10.1002 / \mathrm{nme} .1220$

Obaidat, M.S., P. Nicopolitidis and F. Zarai, 2015. Modeling and Simulation of Computer Networks and Systems: Methodologies and Applications. 1st Edn., Elsevier Science, Amsterdam, ISBN-10: 0128011580, pp: 964.

Ridden, S.J. and B.D. MacArthur, 2012. Cell Fate Regulatory Network. In: New Frontiers of Network Analysis in Systems Biology, Ma'ayan, A. and B.D. MacArthur (Eds.), Springer, ISBN-10: 9400743300 , pp: $15-29$.

Stotzel, C., S. Roblitz and H. Siebert, 2015. Complementing ODE-based system analysis using Boolean networks derived from an Eulerlike transformation. PLOS One, 10: e0140954-e0140954.

DOI: 10.1371 /journal.pone.0140954

Soliman, S. and M. Heiner, 2010. A unique transformation from ordinary differential equations to reaction networks. PLOS One, 5: e14284-e14284. DOI: 10.1371/journal.pone.0014284 
Stai, E., V. Karyotis and S. Papavassiliou, 2015. Analysis and control of information diffusion dictated by user interest in generalized networks. Comput. Soc. Netw., 2: 18-18.

DOI: $10.1186 / \mathrm{s} 40649-015-0025-4$

Tipper, D. and M.K. Sundareshan, 1990. Numerical methods for modeling computer networks under nonstationary conditions. IEEE J. Selected Areas Commun., 8: 1682-1695. DOI: 10.1109/49.62855
Yang, J. and J. Leskovec, 2010. Modeling information diffusion in implicit networks. Proceedings of the IEEE 10th International Conference on Data Mining, Dec. 13-17, IEEE Xplore Press, pp: 599-608.

DOI: $10.1109 /$ ICDM.2010.22 